\title{
Interactions Between Bandwidth Limited CPLs and MMC Based MVDC Supply
}

U. Javaid, A. Christe, F. Freijedo, et al.

This material is posted here with permission of the IEEE. Such permission of the IEEE does not in any way imply IEEE endorsement of any of EPFL's products or services. Internal or personal use of this material is permitted. However, permission to reprint / republish this material for advertising or promotional purposes or for creating new collective works for resale or redistribution must be obtained from the IEEE by writing to pubs-permissions@ieee. org. By choosing to view this document, you agree to all provisions of the copyright laws protecting it. 


\title{
Interactions Between Bandwidth Limited CPLs and MMC Based MVDC Supply
}

\author{
Uzair Javaid, Alexandre Christe, Francisco D. Freijedo and Drazen Dujic \\ Power Electronics Laboratory - PEL \\ École Polytechnique Fédérale de Lausanne - EPFL \\ Station 11, CH-1015 Lausanne \\ uzair.javaid@epfl.ch, alexandre.christe@epfl.ch, francisco.freijedo@epfl.ch,drazen.dujic@epfl.ch
}

\begin{abstract}
With the improvement in the power electronic technologies, medium voltage dc (MVDC) electrical distribution systems are being considered for on-shore and off-shore applications. These future MVDC electrical distribution systems are expected to provide the possibility of easy interfacing of the renewable energy sources, improving the dynamics of the system and also help in reducing the carbon footprint of energy sources. Modular multilevel converters (MMCs) are used in high voltage dc (HVDC) applications and are being considered for MVDC applications as well. In this paper, we present an MVDC electrical distribution system where the source converter is an MMC and the loads exhibit bandwidth limited constant power load (CPL) behaviour. An analysis is carried out on the dynamic interactions between the MMC source converter and CPLs, considering varying distribution cable lengths between the source and the load, the filtering effort at the load end and different loading conditions.
\end{abstract}

\section{INTRODUCTION}

There is an increased interest in usage of the medium voltage dc (MVDC) electrical distribution systems for onshore and off-shore applications[1]-[3]. This shift towards dc systems is a result of the improved power electronic technologies and their wider application in the power systems. This has also increased the penetration of the renewable energy resources in the power system, and has opened the possibilities to use the MVDC electrical distribution system in place of the traditional medium voltage ac (MVAC) electrical distribution systems. The on-shore MVDC electrical distribution systems are expected to increase the flexibility, efficiency and reduce the operational costs of the power system [1]. Advantages of the MVDC electrical distribution systems for off-shore wind farms, which highlights decrease in cost, footprint and filtering effort, and an increase in availability and flexibility, have been reported in [2]. Similarly, for the ship on-board electrical power systems, the MVDC systems provide an opportunity to increase the fuel efficiency and flexibility in the design, and decrease the operational costs of the system and the footprint of the installed equipment [3]. But, all of these possible advantages come with challenges, e.g. lack of standardized dc voltage levels, high power dc-dc converters, rectifiers, MVDC protection components, MVDC cable technology, active and passive filters and energy storage systems [1], [3].

Another major challenge is the stability of the MVDC electrical distribution system due to the presence of the load side inverters that behave as constant power loads (CPLs) [4]-[10].
The impedance modeling of the dc-side of the source and the load converters, for small signal linearization, is addressed in [5], [6]. Additionally, the impedance stability criteria can help in assessing the relative stability utilizing Nyquist and Bode plots [5], [6]. This approach can also be utilized for multiterminal dc systems [7]. Considering these techniques, sourceload interactions for the on-board ship MVDC distribution have been analyzed for different rectifiers and a CPL. Nonlinear control techniques and large signal analysis, to determine and ensure system stability, have also been reported for MVDC electrical systems with buck converters connected to CPLs [9], [10]. Modular multilevel converter (MMC), being a state of the art technology for high voltage dc (HVDC) applications, is also being considered for MVDC applications as it provides higher efficiency, modularity, voltage scalability and reduced filtering effort [1], [11]. MMC as an inverter in a MV back to back drive, highlighting reduced filtering effort, is presented in [12]. The impedance modeling of the MMC, working as a rectifier in marine MVDC electrical distribution systems, has been reported and analyzed for stability in [13]. However, the effects of the distribution cables and the inverter filters have not been analyzed.

In this paper, an MVDC electrical distribution system, of several megawatts (MWs), is analyzed considering an MMC based rectifier supplying bandwidth limited CPLs. Identification of the possible unstable conditions and the dynamic interactions between MMC and CPLs are performed with linearized impedances that are measured by simulations. The dynamic analysis considers the effect of the passive components present on the ac and the dc side of the source converters, cable lengths, filter on the inverter side, impact of the detailed control of the MMC (based on [14]) and the bandwidth limited CPL behavior of the loads. Figures of merit are extracted from the Nyquist plots that provide insight into the design aspects of the future MVDC electrical distribution systems.

This paper is organized as follows: Section II discusses an MVDC electrical distribution system, which is followed by the description of the impedance based stability criteria and the impedance/admittance representation of the source/load subsystems in Section III. The MMC control is explained in Section IV, while, the dynamic stability analysis is presented in Section V. Section VI provides the summary and conclusions. 


\section{MVDC ELECTRICAL DISTRIBUTION SYSTEM}

The MVDC electrical distribution systems for on-shore and off-shore applications are expected to have their respective attributes, e.g. different distribution voltage levels, protection requirements, control structures, load cycles, distribution cables or overhead lines etc. In this paper, a generalized MVDC electrical distribution system, with several MW power levels, is presented and analyzed, as shown in Fig. 1(a). This system consists of an MMC based supply side converter connected to a three phase grid with rated voltage of $6.6 \mathrm{kV}, 50 \mathrm{~Hz}$ frequency and rated at $7 \mathrm{MVA}$. The dc side of the MMC is connected, through a disconnector, to cables supplying four loads through inverters. Each load, in this case, is considered as a motor connected to the MVDC distribution with its own disconnector, inverter and filter.

In general, an MVDC distribution system could be supplied by a diesel generator set, a small wind farm or a solar farm, but for simplicity of the analysis a three phase balanced supply is considered. Additionally, the inverter side can be supplying power to another grid, a data center, driving a large electrical machine or a collection of smaller machines. These inverters are usually based on multilevel voltage source inverter topologies [16], built from high power semiconductors that are usually switched $\leq 1 \mathrm{kHz}$ to reduce switching losses [17]. In any case, a bandwidth limited CPL behavior is characteristic for the load side converters. The distribution system is modeled with a single $\pi$-section for cables, which is fairly accurate for short cable lengths. For this study, a $12 \mathrm{kV}$ XLPE MVAC cable from Brugg Cables is chosen [18]. The system, MMC based supply side converter, cable and load parameters are given in Table I.
TABLE I

SYSTEM PARAMETERS USED FOR THIS STUDY.

\begin{tabular}{|c|c|}
\hline \multicolumn{2}{|l|}{ System Parameters } \\
\hline Rated Apparent Power & $7 \mathrm{MVA}$ \\
\hline Rated Direct Voltage & $10 \mathrm{kV}$ \\
\hline Rated Frequency & $50 \mathrm{~Hz}$ \\
\hline Phase Reactor Inductance & $1 \mathrm{mH}$ \\
\hline Switching frequency for load inverters & $1 \mathrm{kHz}$ \\
\hline \multicolumn{2}{|l|}{ MMC Parameters } \\
\hline Rated Apparent Power & $7 \mathrm{MVA}$ \\
\hline Rated Direct Voltage & $10 \mathrm{kV}$ \\
\hline Number of sub-modules per arm & 10 \\
\hline Switching frequency per sub-module & $315 \mathrm{~Hz}$ \\
\hline Arm capacitance & $0.65 \mathrm{mF}$ \\
\hline Arm Inductance & $1 \mathrm{mH}$ \\
\hline Arm Resistance & $0.1 \mathrm{~m} \Omega$ \\
\hline \multicolumn{2}{|l|}{ Cable Parameters } \\
\hline Line Inductance & $0.347 \mathrm{mH} \mathrm{km}^{-1}$ \\
\hline Line Resistance & $0.089 \Omega \mathrm{km}^{-1}$ \\
\hline Line Capacitance & $0.307 \mu \mathrm{F} \mathrm{km}^{-1}$ \\
\hline \multicolumn{2}{|c|}{ Equivalent Induction Motor Ratings } \\
\hline Rated Power & $6.5 \mathrm{MW}$ \\
\hline Rated Voltage & $6.1 \mathrm{kV}$ \\
\hline Rated Current & $700 \mathrm{~A}$ \\
\hline Rated Frequency & $19 \mathrm{~Hz}$ \\
\hline Rated Speed & $376 \mathrm{rpm}$ \\
\hline Power Factor & 0.92 \\
\hline \multicolumn{2}{|c|}{ Parametric Variations } \\
\hline System Load & $25 \%-100 \%$ \\
\hline Cable Length & $10 \mathrm{~m}-1 \mathrm{~km}$ \\
\hline Inverter Filter & $1 \mathrm{mF}-10 \mathrm{mF}$ \\
\hline
\end{tabular}

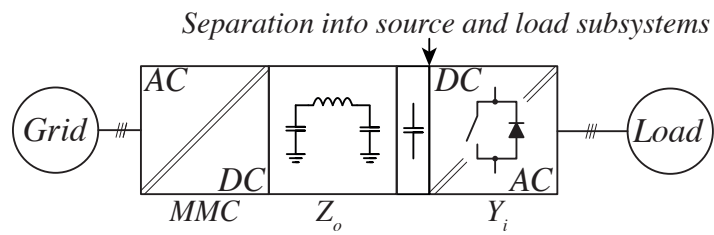

(b)

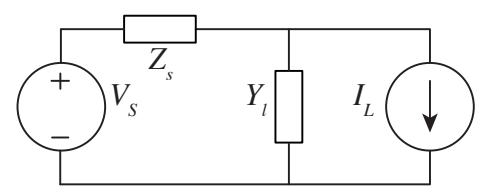

(c)

Fig. 1. MVDC electrical distribution system: (a) Simplified MVDC electrical distribution system. (b) Two-port MVDC distribution system and its appropriate partitioning into source/load subsystems at the arrow [15]. (c) Linearized model for the stability analysis. 


\section{SySTEM DyNAMIC ASSESSMENT AND IMPEDANCE/ADMITTANCE MODELING}

\section{A. Impedance Stability Criteria}

The scope of this paper is to quantify the effect of the passive components present on the ac and the dc side of the source converter, cable inductances, filter on the inverter side and control strategies for both source and load sides (CPL behavior) on the possible resonances in the system and their implications on stability. A complex system is shown in Fig. 1(a). This system can be scaled down to a simplified circuit like Fig. 1(b) ([5], [6]), by assuming that the loads have similar small signal behavior, are connected to the source with same cable lengths and that their individual non-linear effects are neglected. A key feature of Fig. 1(b) is that it shows equivalent source and load. For the sake of generality, this simplified two-port system is suitable to extract figures of merit for different load devices and their interactions with the source. The system of Fig. 1(b), can also be divided into different subsystems, i.e. grid, MMC rectifier, distribution cable (represented as an equivalent $\pi$ - section model), and dc capacitor bank as a source, with the inverter as a load.. For impedance stability analysis, Fig. 1(b) should be further simplified to Fig. 1(c) ([6], [15]). Fig. 1(b) shows the break point considered for the analysis. The minor loop gain, given in (1), is employed to plot the Nyquist contours to assess the stability of the system under consideration.

$$
T(j \omega)=Z_{s}(j \omega) Y_{l}(j \omega)
$$

A complete system model is implemented in PLECS considering detailed control for the MMC rectifier and similarly for the inverter driving a machine. Details of the MMC control are given in the next section. In order to measure the source/load impedance/admittance, a multi-tone perturbation as current/voltage is applied at terminals and the corresponding voltage/current is measured. The ratio of the measured signal to the perturbation signal gives the impedance/admittance [19]

\section{B. Load Admittance}

The lumped load side considers an equivalent induction motor drive that is controlled with field oriented control. The

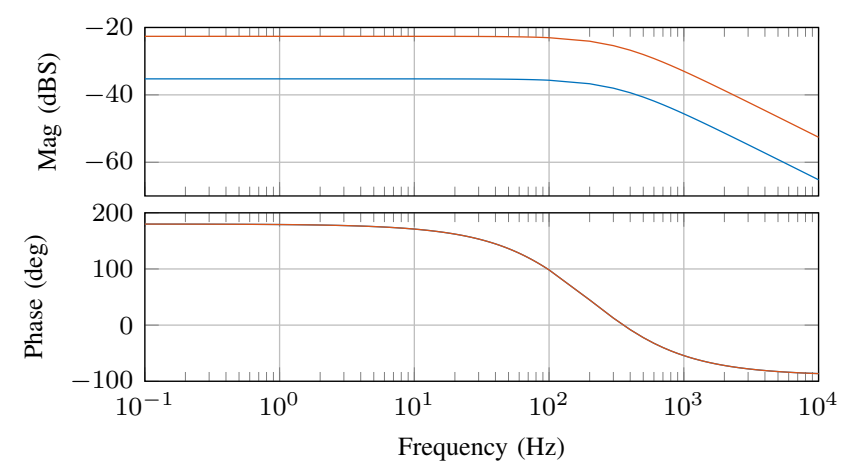

Fig. 2. Load admittance with different loading conditions (Quarter Load and Full Load). parameters of the equivalent induction motor are given in Table I. Load admittance, shown in Fig. 2, is measured for full load, i.e. $100 \%$ of the rated speed and rated torque, and light load, i.e. $100 \%$ of the rated speed and $25 \%$ rated torque. The admittances show a CPL behavior for the majority of the frequency window but changes to an inductive behavior due to the limitations on the current control bandwidth $(100 \mathrm{~Hz})$. Therefore, the motor inductances dominate the load admittance at higher frequencies. This admittance $Y_{l}(j \omega)$ is used for for the small signal analysis in this paper.

\section{Source Impedance}

While there is a large amount of work available on the MMC converter design and operation, there are few works on the MMC dc-side impedance modeling [13], [20]. These works consider a rather simplified implementation of the MMC control and do not consider surrounding equipments on the MVDC side. In this work, we have considered a detailed control of the MMC based on [14], which is explained in the next section. As apparent from Fig. 1(a), the source side impedance consists of three parts: i) grid connected MMC rectifier, ii) cable, and iii) inverter filter. The cable and inverter filter are passive components and play a role in shaping the source impedance, outside the bandwidth of the control of the MMC.

\section{MMC CONTROL STRUCTURE}

The schematics of the MMC rectifier with an average representation of its branch, used in this study, is given in Fig. 3(a). Additionally, $\pi$-section cable model and inverter filter are also given in Fig. 3(a). The control scheme of the MMC converters has a cascaded nature with outer capacitor voltage loop, acting on $V_{d c}, V_{C \Sigma}^{\Sigma}$ and $V_{C \Sigma}^{\Delta}$ and the inner controllers are controlling the circulating currents and line currents on the ac side. The MMC dc side impedance models presented in [13], [20] do not model the effect of the control of $V_{C \Sigma}^{\Sigma}$ and $V_{C \Sigma}^{\Delta}$, but instead consider an arm energy estimator [21]. An explanation of the control structure used in this study is described as follows.

The detailed MMC control structure consists of the capacitor voltage control, circulating current control, grid current control and phase locked loop (PLL), which are shown in Fig. 3(b), Fig. 3(c), Fig. 3(d) and Fig. 3(e), respectively. The capacitor voltage control has three sub-parts namely i) sum $\left(V_{C \Sigma}^{\Sigma}\right)$ of and ii) difference $\left(V_{C \Sigma}^{\Delta}\right)$ of summed capacitor voltages, and iii) dc-bus control $V_{d c}$, as shown in Fig. 3(b). The first control maintains the total energy of the MMC and also ensures the horizontal balancing, while the second control uses the differential energy control for vertical balancing. The $V_{C \Sigma}^{\Sigma}$ voltages are controlled in $\alpha \beta 0$-frame, with PI controllers, so that the horizontal energy balancing $\alpha \beta$-axes and total energy control 0 -axis can be separated [14]. On the other hand, $V_{C \Sigma}^{\Delta}$ is controlled in $a b c$-frame, with a $\mathrm{P}$ controller and matrix $M$ [14]. The matrix $\mathrm{M}$ is based on discussion provided in [22] and its usage ensures the cancellation of the fundamental frequency component of the circulating currents at the DC 


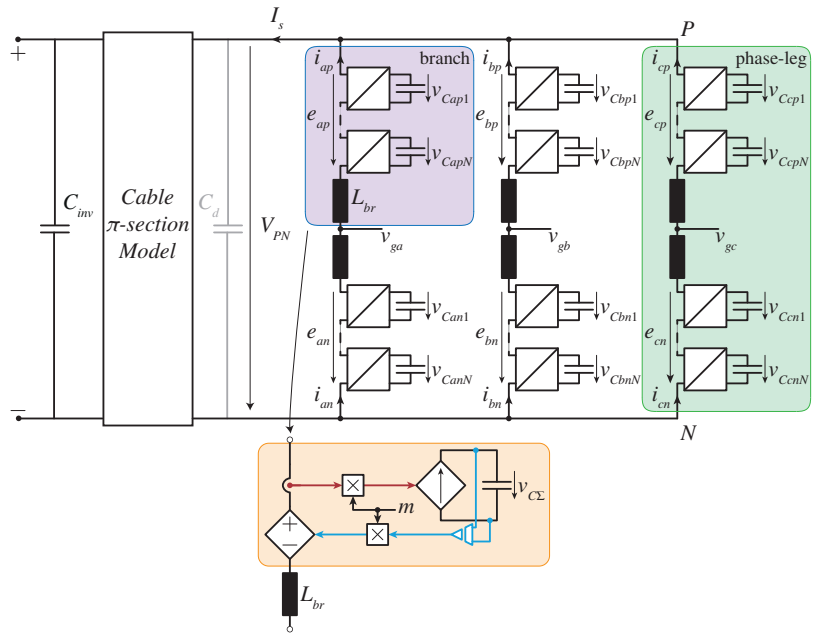

(a)

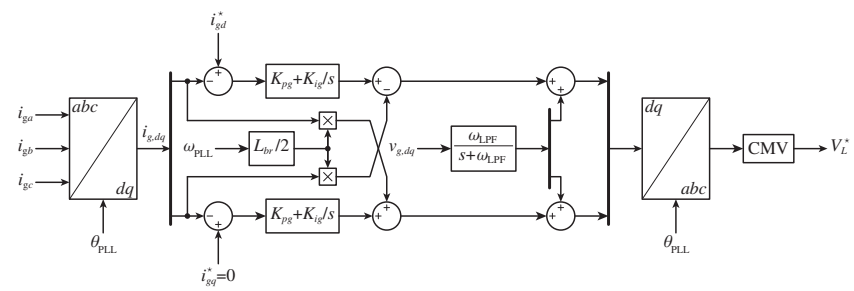

(d)

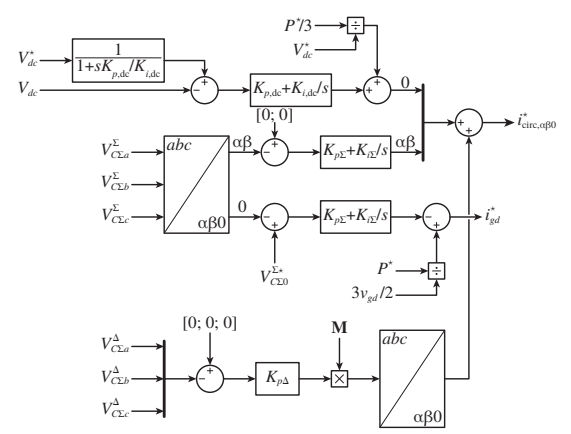

(b)

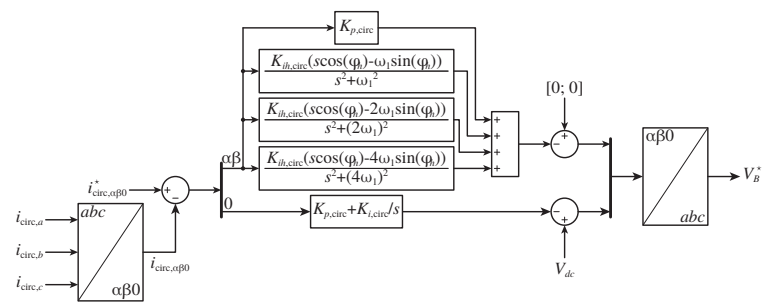

(c)

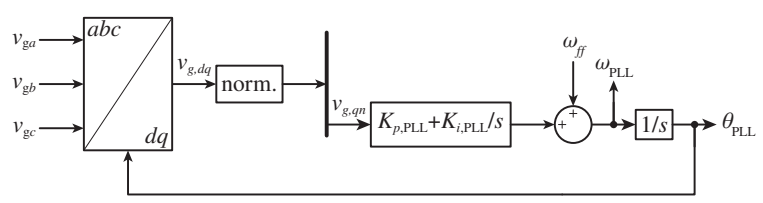

(e)

Fig. 3. Schematics of grid connected MMC based rectifier and its control schemes: (a) MMC based rectifier schematics and branch average model. (b) MMC capacitor voltage controls: de voltage control, and $V_{C}^{\sum}$ and $V_{C}^{\Delta}$ controls for energy balancing. (c) MMC circulating current control inspired by [14]. (d) MMC grid current controller. (e) PLL.

terminals by introducing reactive power flows that do not affect $V_{C \Sigma}^{\Delta}$. Detailed discussions on these control schemes and the required filters are provided in [1], [14]. The dc voltage control is based on [23]. Here, the total energy stored in the internal

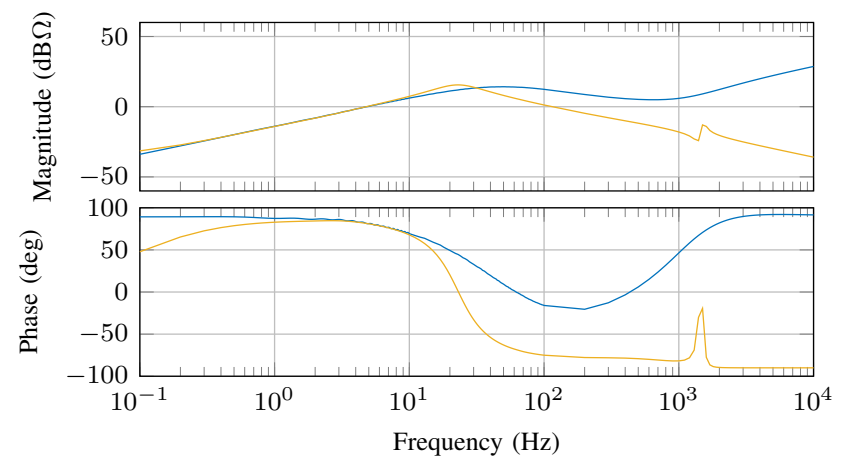

Fig. 4. MMC impedance $\left(Z_{M M C}\right)$ with dc voltage control and source impedance $\left(Z_{s}\right)$ considering a cable length of $100 \mathrm{~m}$ and $C_{i n v}$.
MMC capacitance and equivalent capacitance of the line/cable plays role in maintaining the dc-side voltage. This equivalent capacitance is shown as $C_{d}$ in Fig. 3(a). The plant for tuning dc-side voltage control is, therefore,

$$
C_{e q}=C_{d}+\frac{6 C_{s u b}}{N_{s u b}}
$$

Here, $C_{s u b}$ is the submodule capacitance and $N_{s u b}$ is the number of submodules ${ }^{1}$. The dc-voltage control merges with the total energy control as it provides part of the 0 -axis reference for the circulating current references. The other part of the circulating current references are generated through the differential energy control. The circulating current control, shown in Fig. 3(c), is implemented in $\alpha \beta 0$-frame with PR controllers for $\alpha \beta$-axes and a PI controller for 0 -axis [14]. Here the PR regulators are tuned to fundamental, second and fourth harmonics. The grid current control is implemented in $d q$-frame with PI controllers, including axis decoupling

${ }^{1}$ The value of $C_{d}$ is considered as $0.225 \mathrm{mF}$ for this study, but for small cable lengths it can be neglected $\left(C_{d} \ll C_{\text {branch }}\right)$ and the equivalent internal capacitance of MMC is enough for controlling the dc-side voltage. 


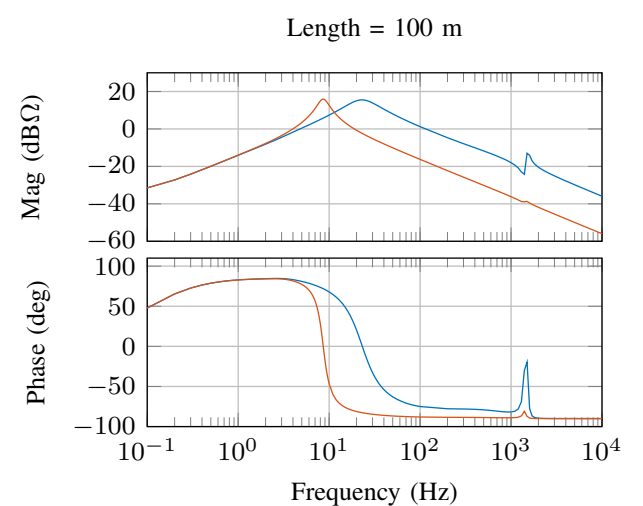

(a)

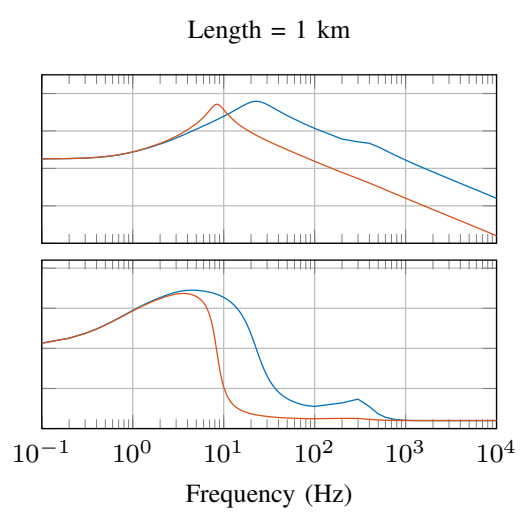

(b)

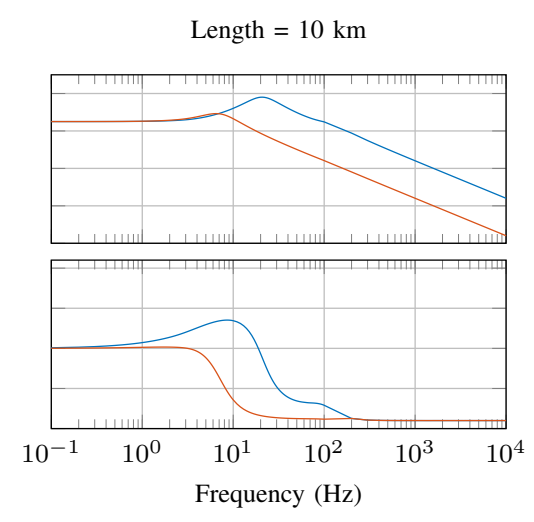

(c)

Fig. 5. Source impedance for different distribution lengths and load filters ( $1 \mathrm{mF}$ and $10 \mathrm{mF})$.

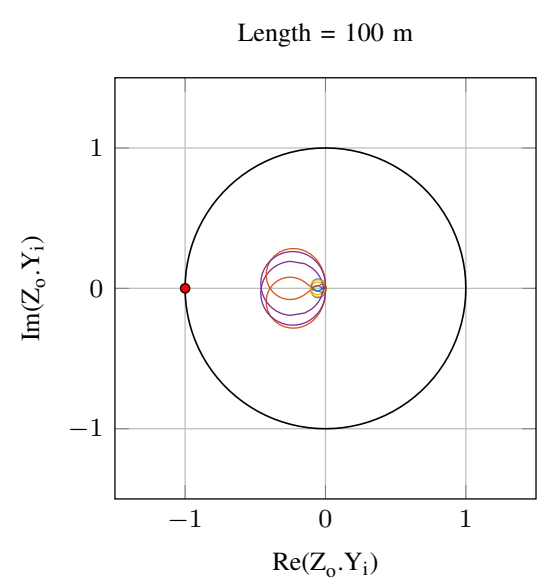

(a)

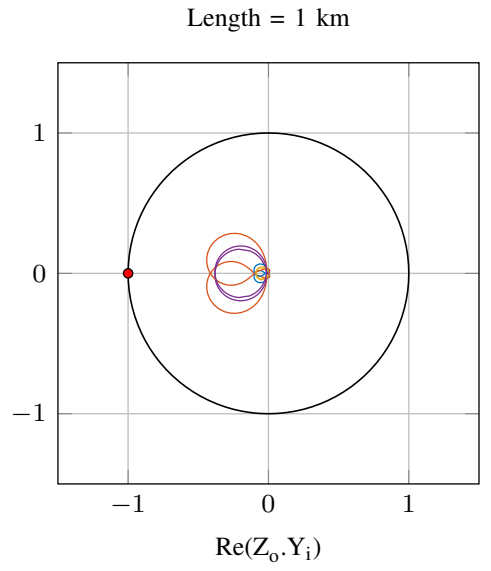

(b)

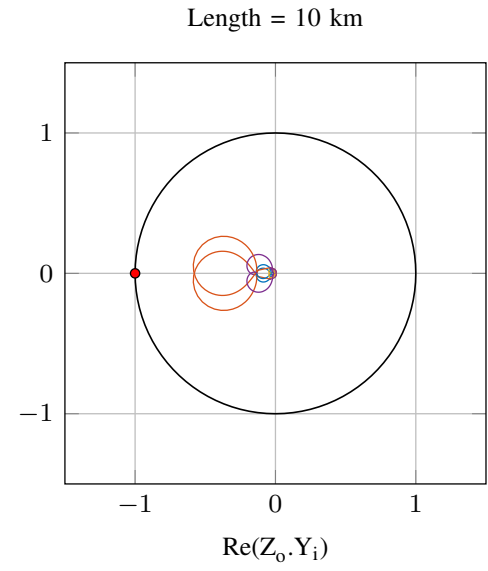

(c)

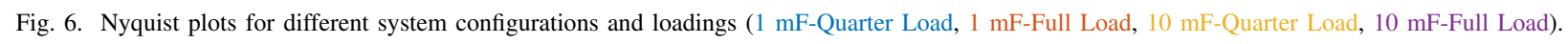

and feed-forward as shown in Fig. 3(d). The PLL, shown in Fig. 3(e), synchronizes the system with the grid frequency. The control parameters of these controllers are given in Table II. All the different controls, being considered here, play a role in shaping $Z_{M M C}$. An analytical expression for $Z_{M M C}$ is not presented here due to the complexity of the control implemented in this study.

In addition to $Z_{M M C}$, the source impedance also has a contribution from cable and inverter filter, therefore, a final expression of the source impedance is:

$$
Z_{s}=\left(Z_{M M C}+Z_{\text {line }}\right) \| Z_{C_{i n v}}
$$

Fig. 4 illustrates the impedance of the MMC with and without the consideration of cable $(100 \mathrm{~m})$ and inverter filter (1 $\mathrm{mF})$. The impedance of the MMC with $V_{d c}$ control, the blue curve shown in Fig. 4, is dominated by voltage control for lower frequencies, i.e. $0.1-100 \mathrm{~Hz}$, but for frequencies higher than $100 \mathrm{~Hz}$ the internal branch inductances dominate the impedance. The addition of line impedance and inverter input
TABLE II

MMC CONTROL PARAMETERS.

\begin{tabular}{|cccc|}
\hline$K_{p, d c}$ & $0.2184 \mathrm{~A} / \mathrm{V}$ & $K_{i, d c}$ & $30.576 \mathrm{As} / \mathrm{V}$ \\
$K_{p, \Sigma}$ & $0.091 \mathrm{~A} / \mathrm{V}$ & $K_{i, \Sigma}$ & $12.74 \mathrm{As} / \mathrm{V}$ \\
$K_{p, \Delta}$ & $0.364 \mathrm{~A} / \mathrm{V}$ & $K_{p, c i r c}$ & $6.72 \mathrm{~V} / \mathrm{A}$ \\
$K_{i, c i r c}$ & $0.672 \mathrm{Vs} / \mathrm{A}$ & $K_{p, g c}$ & $0.84 \mathrm{~V} / \mathrm{A}$ \\
$K_{i, g c}$ & $0.084 \mathrm{Vs} / \mathrm{A}$ & $K_{p, p l l}$ & $91.9 / \mathrm{V}$ \\
$K_{i, p l l}$ & $46 \mathrm{~s} / \mathrm{V}$ & & \\
\hline
\end{tabular}

filter lead to $Z_{s}$. In the high frequency range, the capacitive parts of the line and the inverter filter become dominant in the $Z_{s}$ shape. This is a good behavior in the sense that a low $\left|Z_{s}\right|$ limits the possibility of load/source interactions in at high frequencies. Different source configurations are defined by varying the distribution lengths and the inverter filter, while the parameters of the grid connected MMC rectifier are kept constant. The variations of the parts ii) and iii) of the source impedance are given in Table I. The source impedances 

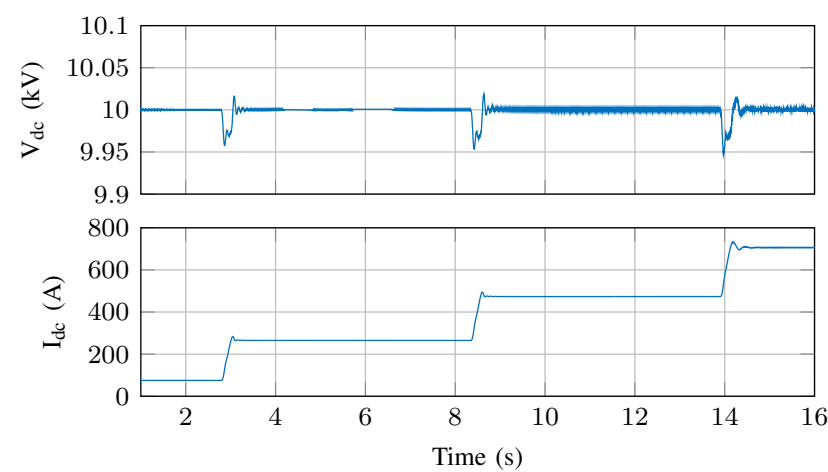

(a)
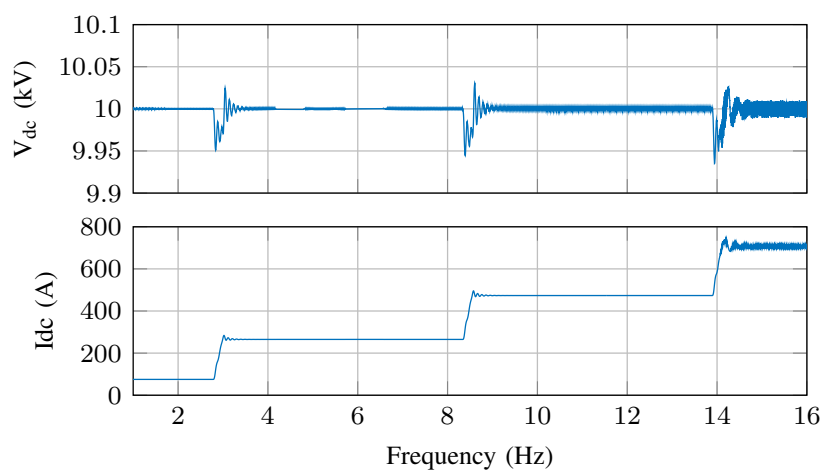

(b)

Fig. 7. Time domain simulation for different system configurations ((a) cable length $100 \mathrm{~m}$ and $C_{\text {inv }}=1 \mathrm{mF}$, (b) cable length $10 \mathrm{~km}$ and $C_{i n v}=1 \mathrm{mF}$ ) and different loading conditions. The load has step changes at $3 \mathrm{~s}, 8.25 \mathrm{~s}$ and $13.9 \mathrm{~s}$ with increments of $25 \%$ till $100 \%$ load is reached.

for the different system configurations are shown in Fig. 5. Here, as discussed earlier, it can be observed that at the lower frequencies, the MMC cascaded control is shaping the impedance and the lower frequency resonance is due to the voltage control of the MMC, and is also impacted, i.e. shifted to slightly lower frequencies by the changes in the inverter filter, as can be seen in Fig. 5. A significantly damped second resonance is between the line inductance and the inverter filter. Higher cable length and filtering effort at the inverter side shifts the second resonance, as well, to the lower frequencies. Another pronounced effect is due to the cable resistance, which at longer cable lengths, damps the resonance in the system and dominates at really low frequencies, as can be observed from Fig. 5(b) and Fig. 5(c).

\section{ImPedance Stability Assessment}

The parametric variations of a possible MVDC system, listed in Table I, are used to evaluate the stability of the two-port equivalent MVDC system, as shown in Fig. 1(b). According to the Nyquist criteria, the system is stable if its Nyquist contour does not encircle the critical point of $(-1,0)$ [15]. A relative stability criterion, based on the inverse of the sensitivity peak $\eta$ is also employed here [24]. This helps in categorizing the system into three different zones i) $\eta>0.5$ high stability, ii) $0.1<\eta<0.5$ low stability, and iii) $\eta<0.1$ unstable. Nyquist contours for three different distribution cable lengths are shown in Fig. 6. All the system configurations, considered in the study, exhibit relatively high stability, i.e. $\eta>0.5$, except for the system configuration with cable length of $10 \mathrm{~km}$ and $C_{i n v}=1 \mathrm{mF}$ that shows low stability i.e $0.1<\eta<0.5$. The relatively low stability is due to the increased inductance in the system and decreased filtering effort at the load side.

Time domain simulation, for the system configuration with $100 \mathrm{~m}$ cable length and inverter filter of $1 \mathrm{mF}$, is presented in Fig. 7(a), while for the system configuration with $10 \mathrm{~km}$ cable length and the same inverter filter, is shown in Fig. 7(b). It can be seen that the predictions made in Fig. 6(a) and Fig. 6(c), for system stability, are fulfilled and the system is stable for different loading conditions.

\section{CONCLUSION}

This paper presents an MVDC electrical distribution system with an MMC based source side converter supplying bandwidth limited CPLs, connected to each other through cables. An analysis is presented that discusses the dynamic interactions between MMC as a MVDC supply and bandwidth limited CPLs. A detailed and realistic control is implemented for the MMC, which includes the dc voltage control, horizontal and vertical energy balancing, circulating current control and line current control. It can be seen that the detailed MMC control, the inverter filter and distribution lengths have a direct impact on shaping the source side impedance. Low filtering at the inverter side and high distribution lengths can slightly decrease the stability of the system. Time domain simulations show that the predictions made from Nyquist criterion are accurate and that MMC as a source side converter ensures a good system stability.

\section{ACKNOWLEDGMENTS}

This work is supported, in part, by the Swiss Commission for Technology and Innovation (CTI) and is part of the Swiss Competence Centers for Energy Research (SCCER) initiative, which focus on Future Swiss Electrical Infrastructure (FURIES), and in part by the Swiss Federal Office of Energy (SFOE) through the research project titled Medium Voltage Direct Current Energy Conversion Technologies and Systems.

\section{REFERENCES}

[1] A. Christe and D. Dujic, "Galvanically isolated modular converter," IET Power Electronics, vol. 9, no. 12, pp. 2318-2328, 2016.

[2] C. Zhan, C. Smith, A. Crane, A. Bullock, and D. Grieve, "Dc transmission and distribution system for a large offshore wind farm," in 9th IET Int. Conf.on AC and DC Power Transmission (ACDC 2010), Oct. 2010, pp. 1-5.

[3] U. Javaid, D. Dujic, and W. van der Merwe, "MVDC marine electrical distribution: Are we ready?" In 41st Annu. Conf. of the IEEE Industrial Electronics Soc. (IECON), Nov. 2015, pp. 000823-000828. 
[4] A. Emadi, A. Khaligh, C. H. Rivetta, and G. A. Williamson, "Constant power loads and negative impedance instability in automotive systems: Definition, modeling, stability, and control of power electronic converters and motor drives," IEEE Trans. Veh. Technol., vol. 55, no. 4, pp. 1112-1125, Jul. 2006

[5] A. Riccobono and E. Santi, "Comprehensive review of stability criteria for dc power distribution systems," IEEE Trans. Ind. Applicat., vol. 50 no. 5 , pp. $3525-3535$, Sep. 2014

[6] S. D. Sudhoff, S. F. Glover, P. T. Lamm, D. H. Schmucker, and D. E. Delisle, "Admittance space stability analysis of power electronic systems," IEEE Trans. Aerosp. Electron. Syst., vol. 36, no. 3, pp. 965 973, Jul. 2000.

[7] J. Sun, "Autonomous local control and stability analysis of multiterminal dc systems," IEEE Trans. Emerg. Sel. Topics Power Electron., vol. 3, no. 4, pp. 1078-1089, Dec. 2015.

[8] U. Javaid, F. D. Freijedo, D. Dujic, and W. van der Merwe, "Dynamic assessment of source-load interactions in marine mvdc distribution," IEEE Trans. Ind. Electron., vol. 64, no. 6, pp. 4372-4381, Jun. 2017

[9] G. Sulligoi, D. Bosich, G. Giadrossi, L. Zhu, M. Cupelli, and A. Monti, "Multiconverter medium voltage dc power systems on ships: Constantpower loads instability solution using linearization via state feedback control," IEEE Trans. Smart Grid, vol. 5, no. 5, pp. 2543-2552, Sep. 2014

[10] M. Cupelli, F. Ponci, G. Sulligoi, A. Vicenzutti, C. S. Edrington, T. ElMezyani, and A. Monti, "Power flow control and network stability in an all-electric ship," Proc. of the IEEE, vol. 103, no. 12, pp. 23552380, Dec. 2015.

[11] P. Blaszczyk, M. Steurer, D. Soto, F. Bogdan, J. Hauer, M. Sloderbeck, and K. Schoder "Modular multilevel converter based test bed for mvdc applications - a case study with a $12 \mathrm{kV}, 5 \mathrm{MW}$ setup," in IEEE International Power Electron. and Motion Control Conf. (PEMC), Sep. 2016, pp. 139-145.

[12] D. Krug, S. Busse, and M. Beuermann, "Complete performance test of mv drive with modular multilevel topology for high power oil gas applications," in Petroleum and Chemical Ind. Tech. Conf. (PCIC), Sep. 2016, pp. 1-6.

[13] R. Mo, Q. Ye, and H. Li, "Dc impedance modeling and stability analysis of modular multilevel converter for mvdc application," in IEEE Energy Conversion Congr. and Expo. (ECCE), Sep. 2016.
[14] N. Cherix, "Functional description and control design of modular multilevel converters-towards energy storage applications for traction networks," PhD thesis, École Polytechnique Fédérale de Lausanne 2015.

[15] R. D. Middlebrook, "Input filter considerations in design and application of switching regulators," IAS Record, 1976.

[16] J. Wahlstroem, D. Dujic, M. A. Luescher, and S. Reist, "High power igct based multilevel inverter," in Proc. of Int. Exhibition and Conf. for Power Electronics, Intelligent Motion, Renewable Energy and Energy Management (PCIM), May 2014, pp. 1-6.

[17] J. K. Steinke, "Control strategy for a three phase ac traction drive with three-level gto pwm inverter," in 19th Annu. IEEE Power Electronics Specialists Conference (PESC), Apr. 1988, 431-438 vol.1.

[18] Brugg cables - mittelspannungskabel - mittelspannungskabel (cu) xkdt 1-leiter ms-polymerkabel 20/12kv, Nov. 2011.

[19] J. Liu, X. Feng, F. C. Lee, and D. Borojevich, "Stability margin monitoring for dc distributed power systems via perturbation approaches,' IEEE Trans. Power Electron., vol. 18, no. 6, pp. 1254-1261, Nov. 2003.

[20] G. Stamatiou, "Analysis of vsc-based hvdc systems," $\mathrm{PhD}$ thesis, Department of Energy and Environement, Chalmers University of Technology, Gothenburg, Sweden, 2016.

[21] A. Antonopoulos, L. Ängquist, L. Harnefors, K. Ilves, and H. P. Nee, "Global asymptotic stability of modular multilevel converters," IEEE Transactions on Industrial Electronics, vol. 61, no. 2, pp. 603-612, Feb. 2014.

[22] P. Münch, D. Görges, M. Izák, and S. Liu, "Integrated current control, energy control and energy balancing of Modular Multilevel Converters," in 36th Annu. Conf. of the IEEE Industrial Electronics Soc. (IECON), Nov. 2010, pp. 150-155.

[23] K. Sharifabadi, L. Harnefors, H.-P. Nee, R. Teodorescu, and S. Norrga Design, Control and Application of Modular Multilevel Converters for HVDC Transmission Systems. John Wiley \& Sons, 2016.

[24] K. J. Astrom and T. Hagglund, PID controllers: Theory, design, and tuning. International Society of Automation, 1995. 